\title{
The pre-modern era of the ISM
}

\author{
John M. Dickey \\ School of Maths and Physics, University of Tasmania, Hobart, TAS 7005, Australia \\ email: john.dickey@utas.edu.au
}

\begin{abstract}
The history of our understanding of the interstellar medium has a pre-modern era and an early-modern era. Several threads show continuity in observational approach and interpretation, reaching from the first decade of the last century to the present. The first 50 years were a period of pioneering work, done mostly by stellar astronomers, who chanced upon the ISM by a strange variety of pathways.
\end{abstract}

Keywords. ISM: general, ISM: evolution, ISM: clouds, ISM: structure, Galaxy: evolution

The study of the interstellar medium (ISM) began around 1900, and the period from then to about 1950 can be considered the pre-modern era. The early-modern era lasts from about 1950 to the late 1970s. In this written version of the talk I have room only to discuss the former epoch.

Astronomers have recognised bright nebulae, regions of extended emission, and dark nebulae, regions where starlight is obscured by foreground clouds, since before the time of Herschel (1833). Around the turn of the last century, photographic techniques had advanced far enough to allow wide-field plates to record both light and dark nebulae (e.g. Draper 1880, Barnard 1903). Barnard in particular took an interest in the large scale structure of the dark nebulae in the constellation Orion that anticipated the mapping work that came some 40 years later. But telescopes of that period were intrinsically small-field, and surface-brightness sensitivity limitations prevented very large scale photographic imaging of the faint structures that Barnard could see and draw.

In 1904 two papers were published that started the two traditional methods of studying the ISM that continued for much of the 20th Century. In Germany, Johannes Hartmann (1904) accidentally discovered a system of spectral lines, notably Ca, toward a binary star whose orbit he was studying. The Ca lines did not change in velocity as the stellar lines moved back and forth due to the Doppler shift of the binary star $\delta$ Orionis. Hartmann correctly interpreted these lines as due to an intervening cloud of gas. Although his conclusion was revolutionary, he spends only one page on it in an 18 page paper on the stellar velocities. He points out that he had seen a similar Ca line in the spectrum of Nova Persei 1901, distinguished from the nova lines both by their different center velocities and their much narrower line-widths.

In the same year, George Comstock (1904) published the results of an extensive survey of stars with distances based on their apparent recoil proper motion due to the Sun's motion relative to the local standard of rest, which had recently been measured. Comstock then computed the expected apparent magnitudes of samples of stars at different distances, and found them to be fainter than his prediction. He ascribes their faintness to an intervening absorbing medium, and he computes a visual obscuration not very different from the modern average of $1.8^{m} \mathrm{kpc}^{-1}$. Although Comstock's conclusion drew a bitter refutation from Jacobus Kapteyn (1904), by 1909 Kepteyn seems to have accepted Comstock's absorbing medium. But many other astronomers refused to accept extinction even as the seemingly obvious explanation for the zone of avoidance (e.g. Shapley 
1918). After the turbulent years of the 1920s, when our paradigm for the universe completely changed, the role of the ISM as a reddening and obscuring medium was generally accepted (reviewed by Trumpler 1930).

Spectroscopic study of the ISM advanced rapidly in the 1930s, with detection of more absorption systems and more species of atoms and the first interstellar molecules: CN, $\mathrm{CH}$ and $\mathrm{CH}^{+}$(Dunham 1937, reviewed by Adams 1949). The first ionization equilibrium calculations to explain diffuse hydrogen emission line regions were done by Ambartsumian (1949) and Strömgren (1939), though Strömgren credits Struve and Elvey (1938) with the first computation of the ionization requirements of what we now call H II regions. At this point the spectroscopic study of the ISM divided into studies of emission lines and absorption lines, which usually sample very different solid angles and often detect atoms in different states of excitation, a distinction that continues today, at all wavelengths.

In the late 1930s and 1940s, working with telescopes at the Burakan and Ambastumani Observatories, Ambartsumian and his collaborators made remarkable progress in quantifying the structure of the dark dust clouds that can be traced by their extinction of the light from background stars and galaxies. Both Chandrasekhar and Münch (1950) and Strömgren (1983) credit his work with changing the paradigm for the structure of the cold ISM from a smooth medium to a population of clouds with low filling factor and high internal density, with about six clouds per kpc on a random line of sight. Ambartsumian's results were later picked up by Spitzer in his seminal textbooks, and the results came to be called Spitzer-standard clouds.

By the early 1950s a flood of new results from both theory and observations of the ISM changed the nature of the field from a curiosity indulged in occasionally by stellar astronomers, to a science in its own right. Before the early 1950s astronomers took stars as a celestial given. After their energy source was understood in detail, and their initial mass function was determined (Salpeter 1955), it became clear that stars had to form continuously through the age of the universe, right to the present. So the role of the ISM as the evolutionary partner of stellar nucleosynthesis in the chemical evolution of galaxies was at last appreciated.

\section{References}

Adams, W. S. 1949, ApJ 109, 354.

Ambartsumian, V. 1932, MNRAS 93, 50.

Barnard, E. E. 1903, ApJ 17, 77.

Chandrasekhar, S. \& Münch, G. 1950, ApJ 112, 380.

Comstock, G. C. 1904, AJ 24, 139.

Dunham, T. 1937, PASP 49, 26.

Draper, H. 1880, Nature 22, 583.

Hartmann, J. 1904, ApJ 19, 268.

Herschel, J. 1833, A Treatise on Astronomy, edited and reproduced by Longmans et al. (Cambridge University Press, 2009) http://messier.seds.org/xtra/similar/herschel.html

Kapteyn, J. C. $1904 A J, 24,115$.

Kapteyn, J. C. 1909 ApJ, 29, 46.

Salpeter, E. E. 1955, ApJ 121, 161.

Shapley, H. 1918, PASP 30, 42.

Strömgren, B. 1939, ApJ 89, 526.

Strömgren, B. 1983, ARAA 21, 1.

Struve, O. and Elvey, C. T. 1938, ApJ 88, 364.

Trumpler, R. J. 1930, PASP 42, 214. 